\title{
The Role of Hypothalamic Tri-Iodothyronine Availability in Seasonal Regulation of Energy Balance and Body Weight
}

\author{
Michelle Murphy ${ }^{1,2}$ and Francis J. P. Ebling ${ }^{1}$ \\ ${ }^{1}$ School of Biomedical Sciences, Queen's Medical Centre, University of Nottingham Medical School, Nottingham NG7 2UH, UK \\ ${ }^{2}$ Division of Nutritional Sciences, School of Biosciences, University of Nottingham, Loughborough LE12 5RD, UK
}

Correspondence should be addressed to Francis J. P. Ebling, fran.ebling@nottingham.ac.uk

Received 11 January 2011; Revised 7 April 2011; Accepted 27 April 2011

Academic Editor: Noriyuki Koibuchi

Copyright (C) 2011 M. Murphy and F. J. P. Ebling. This is an open access article distributed under the Creative Commons Attribution License, which permits unrestricted use, distribution, and reproduction in any medium, provided the original work is properly cited.

\begin{abstract}
Seasonal cycles of body weight provide a natural model system to understand the central control of energy balance. Studies of such cycles in Siberian hamsters suggest that a change in the hypothalamic availability of thyroid hormone is the key determinant of annual weight regulation. Uptake of thyroid hormone into the hypothalamus from the peripheral circulation occurs largely through a specific monocarboxylate transporter expressed by tanycyte cells lining the third ventricle. Tanycytes are the principal brain cell type expressing type II and type III deiodinases, so they control the local concentrations of T4, T3, and inactive metabolites. Type III deiodinase mRNA in tanycytes is photoperiodically upregulated in short photoperiod. This would be expected to reduce the availability of T3 in the hypothalamus by promoting the production of inactive metabolites such as rT3. Experimental microimplantation of T3 directly into the hypothalamus during short-days promotes a long-day phenotype by increasing food intake and body weight without affecting the peripheral thyroid axis. Thus, thyroid hormone exerts anabolic actions within the brain that play a key role in the seasonal regulation of body weight. Understanding the precise actions of thyroid hormone in the brain may identify novel targets for long-term pharmacological manipulation of body weight.
\end{abstract}

\section{Introduction}

Most mammals inhabiting temperate and arctic environments display profound seasonal cycles of body weight reflecting changes in both food intake and energy expenditure. Over the course of a year many animals respond to the changing day length by modifying their behavior and energy metabolism in anticipation of the altered energetic demands of the environment. Thus, seasonal animals typically undergo annual cycles of body fattening in summer when food availability is high followed by loss of adipose tissue as a strategy to survive winter. Many research groups worldwide now exploit seasonal cycles of body weight as a model system in which to understand hypothalamic mechanisms regulating energy balance; in this regard, the Siberian hamster has been used as a model organism since the early 1970s (see Ebling and Barrett [1] for review). Seasonal cycles in energy metabolism, food intake, body weight, and reproductive ability can be driven in this species simply by changing the light dark cycle on which the animals are maintained in the laboratory [2]. Long days of over 12.5 hours of light promote food intake and development of gonads, whereas short days promote weight loss and regression of reproductive organs [3]. Exposure to short (winter) photoperiods induces a state in which hamsters become hypophagic and catabolize abdominal fat reserves such that they lose up to $40 \%$ of total body weight. Additionally, these hamsters molt such that the agouti summer coat is replaced by a white winter coat, and are reproductively inactive compared to hamsters maintained on long (summer) photoperiods which are relatively obese, hyperphagic, and reproductively active. Surprisingly, the neurobiological mechanisms involved in seasonal body weight regulation appear to be quite different to those which regulate short term energy balance, for example, mechanisms underlying the timing of meals and the response to caloric restriction or acute starvation. The aim of this paper is to summarise recent evidence from 
Siberian hamsters and other species demonstrating a key role for changes in hypothalamic thyroid hormone availability in regulating long-term cycles of body weight.

\section{Hypothalamic Regulation of Energy Balance}

The hypothalamus has long been regarded as the pivotal homeostatic structure regulating energy balance, feeding, and satiety. Along with the brainstem it responds to signals from the circulation such as leptin and insulin relating to the condition of energy stores within the body, and also detects levels of energy metabolites such as glucose and fatty acids [4]. In general, weight-loss arising from caloric restriction (energy deficit) results in reduced leptin concentrations, which promotes increased orexigenic gene expression (e.g., NPY, AgRP, and orexin) and decreased anorectic gene expression (e.g., POMC, CART, and TRH) in the hypothalamus. Contrary to prior expectations, a consistent pattern of changes indicative of an animal in a state of energy deficit is not observed in these genes during short dayinduced weight loss in the Siberian hamster. For example, some studies have observed upregulation of CART in the arcuate nucleus in hamsters in short days [5], but other studies have failed to detect this [6] and have not identified seasonal changes in other homeostatic genes such as NPY and orexin [7-9]. A recent study in which AgRP expression was upregulated in the hypothalamus of hamsters using a recombinant adenoassociated viral vector showed that overexpression of AgRP acutely increased food intake and weight gain, but again did not affect photoperiod-regulated seasonal weight loss [10]. Finally, lesions of the arcuate nucleus, the major site of NPY, POMC, and AgRP production do not impede photoperiodic body weight regulation [11]. Collectively, these different experimental approaches suggest that the known hypothalamic "homeostatic" genes are not playing a key role in the generation of seasonal cycles of body weight; thus, we and other groups have embarked on studies to identify novel mechanisms.

\section{Novel Hypothalamic Mechanisms Associated with Seasonal Body Weight Regulation}

Over the last decade, researchers at the Rowett Institute in Aberdeen, Scotland, have carried out studies of differential gene expression in the hypothalami of fat hamsters maintained in long days as compared to lean hamsters kept in short days. Where subtraction hybridization and microarray analyses revealed photoperiod-induced regulation of transcripts, candidate gene expression was confirmed and localized using in situ hybridization. Unexpectedly, clear changes in gene expression were restricted to two structures, the dorsal medial posterior arcuate nucleus (dmpARC), and the ependymal cell layer lining the third ventricle. Within the dmpARC, short photoperiod suppressed expression of genes involved in retinol/retinoic acid signalling including receptors (RAR and RXR $\gamma$ ) and retinol/retinoic acid transporter proteins (cellular retinol binding protein 1 , cellular retinoic acid binding protein 2), and genes involved in histaminergic signalling (H3R), whilst expression of VGF and other secretogranin genes was found to be upregulated $[12,13]$. There is experimental evidence that VGF-derived peptides induce hypophagia and body weight loss, consistent with the hypothesis that increased VGF expression contributes to SD-induced weight loss [14]. Likewise, the decreased histamine 3 receptor expression has been shown to relate to increased electrophysiological activity of the dmpARC in SD [15]. The functional significance of the down-regulation of retinoic acid signalling in this region of the brain has not been determined, perhaps due to the lack of appropriate genetic or pharmacological tools, but, given the known interactions between nuclear retinoic acid receptors and thyroid hormone receptors, this will be of considerable interest.

\section{Photoperiodic Regulation of Hypothalamic Deiodinase Enzyme Gene Expression}

Even more strikingly, a cluster of candidate genes has been identified whose expression is highly localized to the tanycyte cells embedded in the ependymal lining of the ventral third ventricle. In particular, type III deiodinase (DIO3) was found to be dramatically upregulated in the SD state compared to the LD state, peak induction occurring approximately 8 weeks after initial exposure to SD [2]. This corresponds with the time at which hamsters are at their lowest body weight, and testicular regression has occurred [2]. Type II deiodinase (DIO2) was also found to have a similarly restricted expression pattern within the ependymal cell layer. The initial studies in adult hamsters did not detect photoperiodically induced regulation of this gene [2], but, in juvenile [16] and young adult hamsters, there is a modest SD-induced decrease in DIO2 [17]. The functional significance of these findings is that the local availability of $\mathrm{T} 3$ in the hypothalamus is likely to be determined by the relative activity of DIO2, which converts thyroxine (T4) to active $\mathrm{T} 3$ by outer ring deiodination, and DIO3, which reduces activity by converting both $\mathrm{T} 4$ and $\mathrm{T} 3$ to the inactive forms reverse T3 (rT3) and 3'3'-T2, respectively, via inner ring deiodination (Figure 1). Monocarboxylase transporter 8 which is one of the major transporters of thyroid hormone into the brain also has a highly localized expression restricted to tanycytes [17], so it is reasonable to hypothesize that the balance of activities of DIO2 and DIO3 within tanycytes determines the availability of active $\mathrm{T} 3$ within the mediobasal hypothalamus.

\section{Photoperiodic Pathways Controlling Tanycyte Function}

The central pathways by which changes in photoperiod regulate tanycyte function have been clearly identified in mammals, though similar detail is lacking in birds (Figure 1fig1). Melanopsin-containing retinal ganglion cells in the mammalian retina integrate changes in luminescence and regulate the activity of the suprachiasmatic nucleus such that 


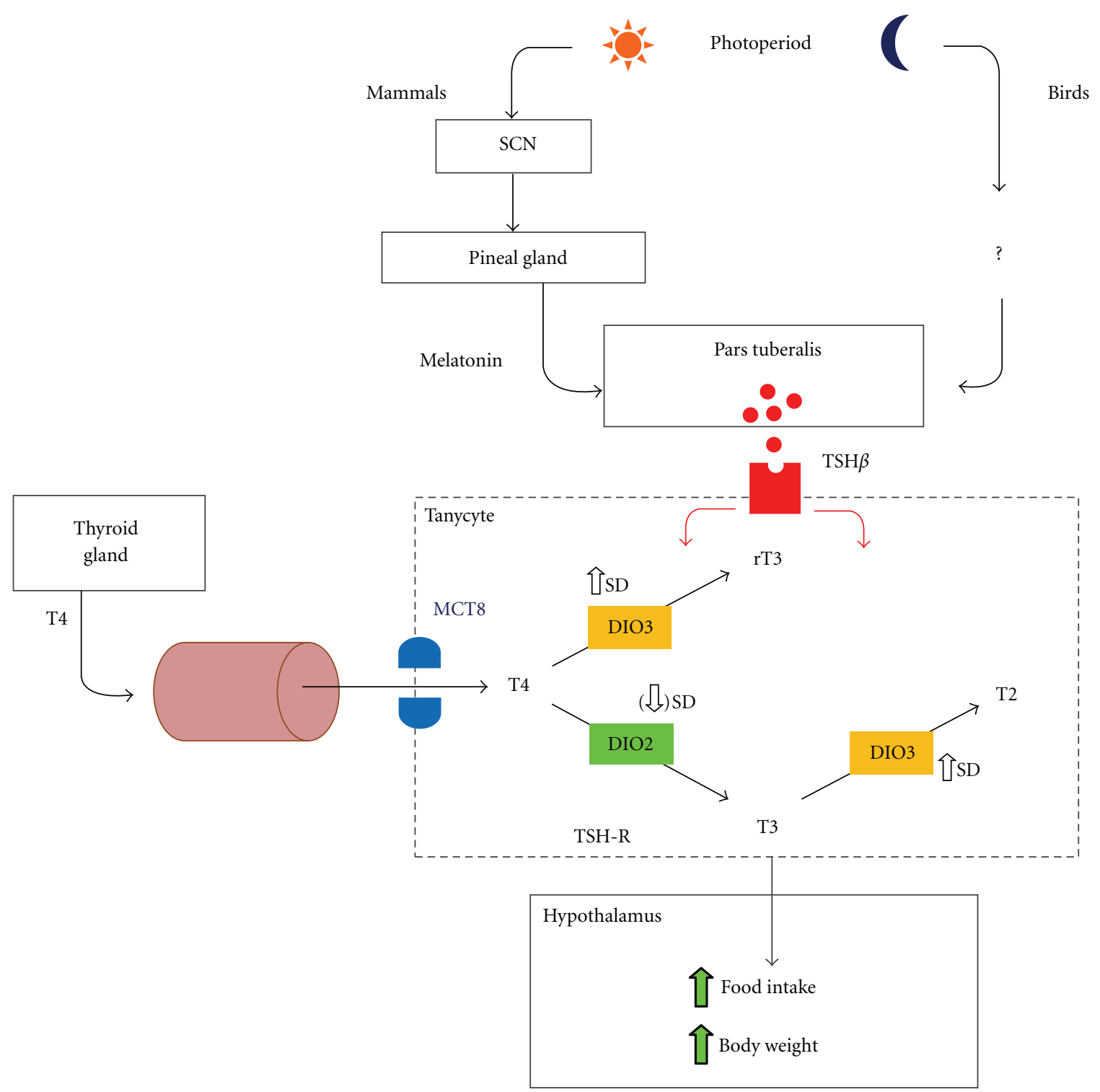

FIGURE 1: Schematic representation of the influence of photoperiod on deiodinase enzyme gene expression in the hypothalamus. In mammals, photoperiodic information detected by the retina is relayed via the suprachiasmatic nucleus (SCN) to the pineal gland, where the nocturnal secretion of melatonin signals directly to the pars tuberalis. In birds, photoperiodic signalling is not dependent on this pinealmelatonin pathway. Across both vertebrate groups, long photoperiods promote TSH $\beta$ production by the pars tuberalis which maintains DIO2 expression in tanycytes. T4 is taken up from the circulation via MCT8 transporters and is converted by type 2 deiodinase (DIO2) to T3 which exerts local anabolic actions within the mediobasal hypothalamus. In short photoperiods (SD) the long nocturnal duration of melatonin reduces TSH $\beta$ production, expression of type 3 deiodinase (DIO3) is upregulated, thus, inactive metabolites of T4 are produced resulting in a catabolic state.

sympathetic control of melatonin production of the pineal gland is upregulated during the dark phase. As photoperiod changes annually, so does the nocturnal duration of melatonin production, thus melatonin can be considered to provide a neurochemical index of night length. The pars tuberalis, a component of the pituitary stalk which apposes the median eminence of the hypothalamus, contains a strikingly high density of melatonin receptors in all species studied to date [18]. A number of melatonin-regulated genes have been identified in this tissue, but changes in expression of TSH $\beta$ are particularly striking, being hugely upregulated in sheep by long days/short melatonin durations [19]. TSH $\beta$ is similarly upregulated in quail by long days [20], suggesting that photoperiodic control of TSH $\beta$ in the pars tuberalis was a trait of ancestral vertebrates, though this is not a melatonindependent process in birds [20]. Recent studies also confirm that tanycytes in sheep express the TSH-receptor, and that TSH infusion in sheep and quail can upregulate DIO2 expression, supporting the view that $\mathrm{TSH} \beta$ is a key paracrine signal released by the pars tuberalis which acts locally to effect photoperiodic changes in tanycyte gene expression and function $[19,20]$. 


\section{Manipulation of Hypothalamic T3 Concentrations Affects Energy Metabolism}

It has been appreciated for many years that experimental manipulation of peripheral thyroid hormone concentrations disrupts seasonal cycles of reproductive activity in a variety of avian and mammalian species, as reviewed by Bechtold and Loudon [21]. However, the physiological mechanism underlying such observations has only become clear with the recent observations that photoperiod-regulated changes in DIO2 and DIO3 expression in tanycytes generate natural changes in thyroid hormone availability in the hypothalamus. Our observations on deiodinase mRNA levels in tanycytes led us to hypothesize that hamsters in SD, where levels of DIO3 are at their highest, would have reduced concentrations of T3 in the hypothalamus. We have therefore conducted a number of studies to determine the role of this change in hypothalamic T3 availability in generating seasonal cycles of body weight.

Previous studies in the sheep demonstrated that placement of microimplants releasing T3 directly into the hypothalamus could reverse the cessation of seasonal reproductive cycles induced by thyroidectomy [22]. The release of thyroid hormone from such implants is confined to the locality of the hypothalamus and does not appear in the systemic circulation [22]. We adopted a similar technology to deliver T3 directly into the hypothalamus of hamsters, these implants contained approximately $10 \mu \mathrm{g}$ of hormone and released 100 pg T3 per day when tested in vitro. In our initial study, microimplants were placed bilaterally into hamsters maintained in long days of 16 hours light: 8 hours dark [2]. These T3-implanted hamsters were then transferred to short days comprising 8 hours light: 16 hours dark. Whereas hamsters bearing sham implants in their hypothalamus displayed the expected short-day response of reduced food intake, body weight loss (Figure 2), and testicular regression, body weight gradually increased in hamsters receiving hypothalamic implants of T3, comparable to a third sham-implanted group of hamsters maintained throughout in long days (Figure 2, [2]). Likewise, epididymal fat pad weight was significantly reduced in sham-implanted animals in SD, but the hypothalamic T3 implants completely prevented this catabolic process (Figure 2) [2].However, animals in the study were still photo-responsive, since the pelage of all hamsters in SD molted to a white winter state, suggesting that seasonal pelage change is not under the control of hypothalamic T3. The winter molt in hamsters results from a SD-induced decrease in prolactin secretion from the pituitary gland [23]. The observation that molting was unaffected by manipulation of hypothalamic T3 levels is consistent with the experimental evidence from sheep with pituitary-hypothalamic disconnection that melatonin acts via the pars tuberalis to regulate directly seasonal cycles of prolactin secretion from the anterior pituitary [24].

We have more recently conducted a second series of studies where hamsters were initially exposed to short days to induce weight loss, and after, 11-week exposure to short days, hamsters received intrahypothalamic T3 implants as described above. Consistent with the initial study, the implants induced a long day phenotype, including increased appetite, increased body weight, and premature recrudescence of the reproductive axis [25]. The induction of this anabolic state was even more rapid than in a control group of hamsters that was transferred at week 11 to long days. Another experiment was conducted in hamsters which had been surgically implanted with an intra-abdominal radiotelemetry device which measures and transmits core body temperature to an external receiver. This allowed us to monitor the presence of torpor, defined as a reduction in body temperature to near ambient temperature which results from a decrease in metabolic rate. It is a mechanism used by seasonal animals to reduce energy requirements and increase their chance of survival when conditions become harsh in winter. The timing of individual torpor bouts follows a circadian pattern and occurs during the hamster's resting period in the light phase. Under artificial photoperiod, torpor has been observed in Siberian hamsters after about 10 weeks of exposure to SD [26]. We observed that shamimplanted hamsters underwent frequent bouts of torpor from week 12 in SD until at least the end of the study after 24 weeks of exposure to SD. However, in hamsters receiving intrahypothalamic $\mathrm{T} 3$ implants, we very rarely observed bouts of torpor, again indicating that increasing $\mathrm{T} 3$ concentrations within the hypothalamus induced a long-day phenotype [25]. As previous studies have shown that high circulating concentrations of testosterone prevent the onset of torpor [27], one might anticipate that the mechanism of actions of $\mathrm{T} 3$ would be to reactivate the reproductive axis, thereby increasing testosterone production. We therefore carried out this study in castrated hamsters, yet still found a profound blockade of SD-induced torpor. This indicates that the actions of T3 are independent of reproductive status, and are true central anabolic actions.

\section{Regulation of Hypothalamic T3 Availability in Other Seasonal Models}

Regulation of deiodinase gene expression has been observed in a wide range of seasonal mammals and birds in addition to the Siberian hamster, though in most species it is DIO2 rather than DIO3 that shows a greater degree of photoperiodic regulation. Thus, long-day induced upregulation of DIO2 has been reported in sheep, Japanese quail, Syrian hamsters, and photoperiodic strains of mice and rats (see [28] for review). However, a common feature across these species is that the predicted effect of altered deiodinase gene expression is greater availability of T3 in the long-day state as compared to the short-day state. The relevance of increased T3 availability in long days of spring-summer to reproduction is not yet clear because seasonal cycles of reproduction vary greatly in their timing between different species. For example, whereas long days promote reproductive activity in many species with short gestation periods, for example, voles, mice, shrews and lagomorphs, they terminate reproductive activity in species with longer gestation periods, for example, sheep, goats, and red deer. However, almost all terrestrial species display photoperiodically programmed anabolic responses (increased food intake, 


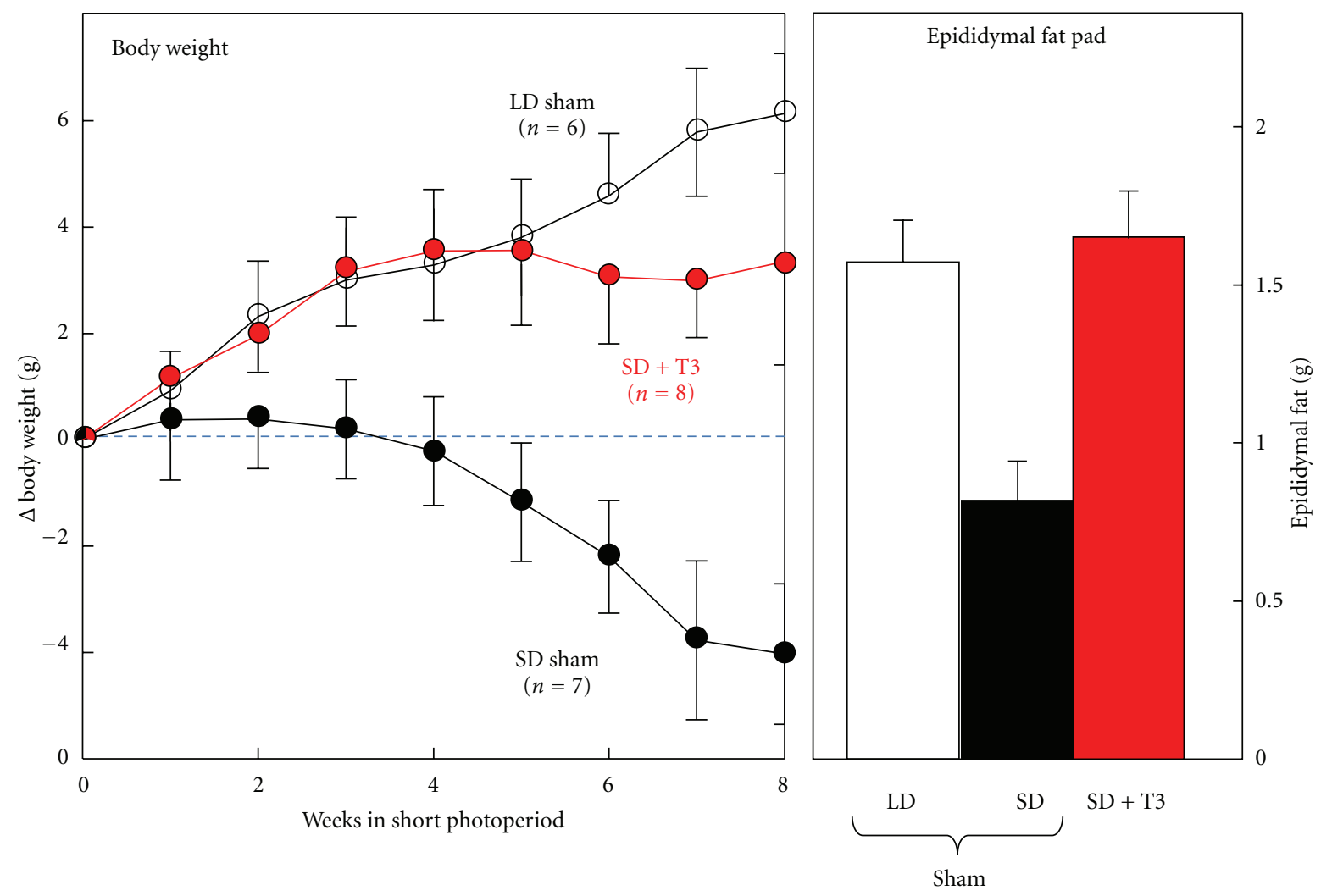

FIGURE 2: Effects of intrahypothalamic microimplants releasing tri-iodotyronine (T3) on body weight (left) and epididymal weight (right) in hamsters transferred to short days (SD+T3). Hamsters receiving sham implants were also transferred to short days (SD sham) or maintained in long days (LD sham). Note that SD induced weight loss, reflecting a reduction of white fat depots as compared to hamsters maintained in LD, but that implants which increased hypothalamic T3 concentrations blocked this SD-induced catabolic response. Modified from Barrett et al. [2].

body fat deposition) in long days. The commonality of anabolic responses associated with high hypothalamic T3 concentrations across so many avian and mammalian species indicates a long evolutionary history [19] and reemphasises the importance of this aspect of thyroid hormone action in understanding control of body weight.

\section{Hypothalamic Actions of Thyroid Hormone}

Our recent understanding that high levels of $\mathrm{T} 3$ in the hypothalamus promote an anabolic state, whereas low levels facilitate a catabolic state may initially seem paradoxical given our clear understanding that in the periphery increased T3 concentrations promote catabolic responses. For example, thermogenesis increases two fold with T3 administration, hence increasing metabolic rate [29], and a number of peripheral targets of T3 have been identified that cause increased energy expenditure during cold exposure in winter, for example, induction of adaptive thermogenesis occurs in brown adipose tissue and perhaps skeletal muscle [30-32]. T3 is also associated with the regulation of muscle phenotype, with a reduction of approximately $4 \%$ of slow fibres in rat hind limb muscles after treatment with T3 [33]. The increased metabolic rate produced by peripheral administration of T3 might also be explained by the higher percentage of type II glycolytic muscle fibres [29]. However, the observation that increased central T3 in hamsters promotes anabolic responses is not without precedent. For example, Kong et al. [34] showed that central administration of T3 in Wistar rats stimulated food intake, though two other studies of hypothalamic administration of T3 in rats did not identify effects on appetite $[35,36]$. However, these studies do provide evidence of other actions of T3 within the hypothalamus; Klieverik et al. [35] demonstrated that intracerebroventricular administration of $\mathrm{T} 3$ or infusion directly into the paraventricular nucleus resulted in increased hepatic glucose production via stimulation of sympathetic innervation of the liver. Likewise, López et al. [36] demonstrated that intracerebroventricular (icv) infusion of T3 reduced phosphorylation of AMPkinase in the ventromedial nucleus which was associated with increased sympathetic outflow and activation of thermogenesis in brown fat. The question arises as to whether the metabolic responses to increased central T3 availability in our own studies and those reviewed above reflect, at least in part, the stimulation of a feedback loop such that suppression of the central control of the HPT axis occurs. However, we have not observed clear differences in TRH mRNA abundance in hypothalamic regions in the $\mathrm{LD}$ and $\mathrm{SD}$ state when hypothalamic $\mathrm{T} 3$ 
availability would be predicted to differ [37], and both Klieverik et al. and López et al. [35, 36] report that icv T3 infusion does not alter circulating T3 concentrations, so there is no evidence to support the conjecture that the central effects of T3 administration are indirect effects via feedback suppression of the hypothalamo-pituitary-thyroid axis.

It should also be noted that the glucoregulatory actions of T3 reported by Klieverik et al. [35] are rapid actions, which the authors therefore attribute to nontranscriptional mechanisms. The responses that we have observed are relatively long-term, so likely represent genomic effects of thyroid-receptor activation. We favour the hypothesis that T3 exerts its seasonal hypothalamic actions via long-term structural and functional plasticity rather than by acutely altering the synthesis and secretion of TRH or other neuropeptides. Whereas TR $\alpha$ is expressed almost ubiquitously in the adult brain, $\operatorname{TR} \beta 1$ and $\operatorname{TR} \beta 2$ are predominantly found in the mediobasal and posterior hypothalamus of the hamster [2], consistent with the hypothesis that T3 exerts genomic actions in these regulatory regions. It is clear that thyroid hormone plays a crucial role in the initial development of the brain [38], so perhaps seasonal cycles of growth, body weight, and reproduction are a recapitulation of these early developmental mechanisms. The cessation of reproductive function in SD has been compared to a reversal of puberty, [28] so likewise the reduction of food intake and loss of body weight might be considered to reflect an arrest of development due to withdrawal of thyroid hormone availability. Other feedback signals have been demonstrated to exert structural effects in the hypothalamus, for example, leptin clearly promotes neurite outgrowth of developing NPY and POMC neurons [39]. Moreover, there is now clear evidence for neurogenesis in the adult hypothalamus [40], demonstrating that this part of the brain retains the potential for plasticity. As such, identifying the precise cellular and molecular mechanisms of thyroid hormone action in the brain may provide insight into mechanisms that would be effective targets for long-term control of appetite and body weight.

\section{Conclusion}

It is clear that hypothalamic T3 availability plays a major role in seasonal cycles of body weight and energy balance. Central T3 levels are regulated quite separately from circulating concentrations; transport into the brain is via specific transporters principally expressed in tanycyte cells, and biologically active concentrations are dependent on the relative activity of the different deiodinase enzymes also present in tanycytes. The cellular mechanisms of action of T3 within the hypothalamus which ultimately generate the annual cycles in food intake and body weight are unknown, but possibly involve plasticity of structure and connectivity.

\section{Acknowledgments}

The authors' work was funded by a BBSRC (UK) Project Grant BB/E020437/1 and by a Targeted BBSRC Studentship in Integrative Mammalian Physiology.

\section{References}

[1] F. J. P. Ebling and P. Barrett, "The regulation of seasonal changes in food intake and body weight," Journal of Neuroendocrinology, vol. 20, no. 6, pp. 827-833, 2008.

[2] P. Barrett, F. J. P. Ebling, S. Schuhler et al., "Hypothalamic thyroid hormone catabolism acts as a gatekeeper for the seasonal control of body weight and reproduction," Endocrinology, vol. 148, no. 8, pp. 3608-3617, 2007.

[3] P. J. Morgan, A. W. Ross, J. G. Mercer, and P. Barrett, "What can we learn from seasonal animals about the regulation of energy balance?" in Proceedings of the 24th International Summer School of Brain Research, Elsevier Science, Amsterdam, Netherlands, 2005.

[4] S. Stanley, K. Wynne, B. McGowan, and S. Bloom, "Hormonal regulation of food intake," Physiological Reviews, vol. 85, no. 4, pp. 1131-1158, 2005.

[5] J. G. Mercer, C. Ellis, K. M. Moar, T. J. Logie, P. J. Morgan, and C. L. Adam, "Early regulation of hypothalamic arcuate nucleus CART gene expression by short photoperiod in the Siberian hamster," Regulatory Peptides, vol. 111, no. 1-3, pp. 129-136, 2003.

[6] A. J. Robson, K. Rousseau, A. S. I. Loudon, and F. J. P. Ebling, "Cocaine and amphetamine-regulated transcript mRNA regulation in the hypothalamus in lean and obese rodents," Journal of Neuroendocrinology, vol. 14, no. 9, pp. 697-709, 2002.

[7] Z. Atcha, F. R. A. Cagampang, J. A. Stirland et al., "Leptin acts on metabolism in a photoperiod-dependent manner, but has no effect on reproductive function in the seasonally breeding Siberian hamster (Phodopus sungorus)," Endocrinology, vol. 141, no. 11, pp. 4128-4135, 2000.

[8] A. B. Reddy, A. S. Cronin, H. Ford, and F. J. P. Ebling, "Seasonal regulation of food intake and body weight in the male Siberian hamster: studies of hypothalamic orexin (hypocretin), neuropeptide Y (NPY) and pro-opiomelanocortin (POMC)," European Journal of Neuroscience, vol. 11, no. 9, pp. 32553264, 1999.

[9] J. G. Mercer, B. C. Lawrence, K. M. Moar, T. Atkinson, and P. Barrett, "Short-day weight loss and effect of food deprivation on hypothalamic NPY and CRF mRNA in Djungarian hamsters," American Journal of Physiology, vol. 273, no. 2, pp. R768-R776, 1997.

[10] P. H. Jethwa, A. Warner, M. J. Fowler et al., "Short-days induce weight loss in Siberian hamsters despite overexpression of the agouti-related peptide gene," Journal of Neuroendocrinology, vol. 22, no. 6, pp. 564-575, 2010.

[11] F. J. P. Ebling, O. J. Arthurs, B. W. Turney, and A. S. Cronin, "Seasonal neuroendocrine rhythms in the male Siberian hamster persist after monosodium glutamate-induced lesions of the arcuate nucleus in the neonatal period," Journal of Neuroendocrinology, vol. 10, no. 9, pp. 701-712, 1998.

[12] K. N. Nilaweera, Z. A. Archer, G. Campbell et al., "Photoperiod regulates genes encoding melanocortin 3 and serotonin receptors and secretogranins in the dorsomedial posterior arcuate of the Siberian hamster," Journal of Neuroendocrinology, vol. 21, no. 2, pp. 123-131, 2009.

[13] A. W. Ross, C. A. Webster, J. G. Mercer et al., "Photoperiodic regulation of hypothalamic retinoid signaling: association of retinoid X receptor $\gamma$ with body weight," Endocrinology, vol. 145, no. 1, pp. 13-20, 2004.

[14] P. H. Jethwa, A. Warner, K. N. Nilaweera et al., "VGF-derived peptide, TLQP-21, regulates food intake and body weight in 
Siberian hamsters," Endocrinology, vol. 148, no. 8, pp. 4044 4055, 2007.

[15] P. Barrett, M. van den Top, D. Wilson et al., "Short photoperiod-induced decrease of histamine $\mathrm{H} 3$ receptors facilitates activation of hypothalamic neurons in the Siberian hamster," Endocrinology, vol. 150, no. 8, pp. 3655-3663, 2009.

[16] M. Watanabe, S. Yasuo, T. Watanabe et al., "Photoperiodic regulation of type 2 deiodinase gene in djungarian hamster: possible homologies between avian and mammalian photoperiodic regulation of reproduction," Endocrinology, vol. 145, no. 4, pp. 1546-1549, 2004.

[17] A. Herwig, D. Wilson, T. J. Logie et al., "Photoperiod and acute energy deficits interact on components of the thyroid hormone system in hypothalamic tanycytes of the Siberian hamster," American Journal of Physiology, vol. 296, no. 5, pp. R1307-R1315, 2009.

[18] P. J. Morgan, P. Barrett, H. E. Howell, and R. Helliwell, "Melatonin receptors: localization, molecular pharmacology and physiological significance," Neurochemistry International, vol. 24, no. 2, pp. 101-146, 1994.

[19] E. A. Hanon, G. A. Lincoln, J. M. Fustin et al., "Ancestral TSH mechanism signals summer in a photoperiodic mammal," Current Biology, vol. 18, no. 15, pp. 1147-1152, 2008.

[20] N. Nakao, H. Ono, T. Yamamura et al., "Thyrotrophin in the pars tuberalis triggers photoperiodic response," Nature, vol. 452, no. 7185, pp. 317-322, 2008.

[21] D. A. Bechtold and A. S. I. Loudon, "Hypothalamic thyroid hormones: mediators of seasonal physiology," Endocrinology, vol. 148, no. 8, pp. 3605-3607, 2007.

[22] G. M. Anderson, S. L. Hardy, M. Valent, H. J. Billings, J. M. Connors, and R. L. Goodman, "Evidence that thyroid hormones act in the ventromedial preoptic area and the premammillary region of the brain to allow the termination of the breeding season in the ewe," Endocrinology, vol. 144, no. 7, pp. 2892-2901, 2003.

[23] M. J. Duncan and B. D. Goldman, "Hormonal regulation of the annual pelage color cycle in the Djungarian hamster, Phodopus sungorus. II. role of prolactin," Journal of Experimental Zoology, vol. 230, no. 1, pp. 97-103, 1984.

[24] G. A. Lincoln and I. J. Clarke, "Photoperiodically-induced cycles in the secretion of prolactin in hypothalamo-pituitary disconnected rams: evidence for translation of the melatonin signal in the pituitary gland," Journal of Neuroendocrinology, vol. 6, no. 3, pp. 251-260, 1994.

[25] M. Murphy, J. Brameld, and F. J. P. Ebling, "The effect of thyroid hormone on torpor in the Siberian hamster (Phodopus sungorus)," in Proceedings of the 7th International Congress of Neuroendocrinology, Publications des universites de Rouen et du Havre, 2010.

[26] T. J. Bartness, J. A. Elliott, and B. D. Goldman, "Control of torpor and body weight patterns by a seasonal timer in Siberian hamsters," American Journal of Physiology, vol. 257, no. 1, pp. R142-R149, 1989.

[27] N. F. Ruby, R. J. Nelson, P. Licht, and I. Zucker, "Prolactin and testosterone inhibit torpor in Siberian hamsters," American Journal of Physiology, vol. 264, no. 1, pp. R123-R128, 1993.

[28] F. J. P. Ebling, "Photoperiodic regulation of puberty in seasonal species," Molecular and Cellular Endocrinology, vol. 324, no. 12, pp. 95-101, 2010.

[29] M. Krotkiewski, "Thyroid hormones in the pathogenesis and treatment of obesity," European Journal of Pharmacology, vol. 440, no. 2-3, pp. 85-98, 2002.

[30] A. C. Bianco, A. L. Maia, W. S. da Silva, and M. A. Christoffolete, "Adaptive activation of thyroid hormone and energy expenditure," Bioscience Reports, vol. 25, no. 3-4, pp. 191-208, 2005.

[31] L. A. de Jesus, S. D. Carvalho, M. O. Ribeiro et al., "The type 2 iodothyronine deiodinase is essential for adaptive thermogenesis in brown adipose tissue," Journal of Clinical Investigation, vol. 108, no. 9, pp. 1379-1385, 2001.

[32] N. Potrović, G. Cvijić, and V. Davidović, "Thyroxine and triiodothyronine differently affect uncoupling protein-1 content and antioxidant enzyme activities in rat interscapular brown adipose tissue," Journal of Endocrinology, vol. 176, no. 1, pp. 31-38, 2003.

[33] A. Vadászová, G. Zachařová, K. Macháčová, I. Jirmanová, and T. Soukup, "Influence of thyroid status on the differentiation of slow and fast muscle phenotypes," Physiological Research, vol. 53, supplement 1, pp. S57-S61, 2004.

[34] W. M. Kong, N. M. Martin, K. L. Smith et al., "Triiodothyronine stimulates food intake via the hypothalamic ventromedial nucleus independent of changes in energy expenditure," Endocrinology, vol. 145, no. 11, pp. 5252-5258, 2004.

[35] L. P. Klieverik, S. F. Janssen, A. van Riel et al., "Thyroid hormone modulates glucose production via a sympathetic pathway from the hypothalamic paraventricular nucleus to the liver," Proceedings of the National Academy of Sciences of the United States of America, vol. 106, no. 14, pp. 5966-5971, 2009.

[36] M. López, L. Varela, M. J. Vázquez et al., "Hypothalamic AMPK and fatty acid metabolism mediate thyroid regulation of energy balance," Nature Medicine, vol. 16, no. 9, pp. 1001$1008,2010$.

[37] F. J. P. Ebling, D. Wilson, J. Wood et al., "The thyrotropinreleasing hormone secretory system in the hypothalamus of the Siberian hamster in long and short photoperiods," Journal of Neuroendocrinology, vol. 20, no. 5, pp. 576-586, 2008.

[38] J. Bernal, "Thyroid hormone receptors in brain development and function," Nature Clinical Practice Endocrinology and Metabolism, vol. 3, no. 3, pp. 249-259, 2007.

[39] S. G. Bouret, S. J. Draper, and R. B. Simerly, "Trophic action of leptin on hypothalamic neurons that regulate feeding," Science, vol. 304, no. 5667, pp. 108-110, 2004.

[40] M. V. Kokoeva, H. L. Yin, and J. S. Flier, "Evidence for constitutive neural cell proliferation in the adult murine hypothalamus," Journal of Comparative Neurology, vol. 505, no. 2, pp. 209-220, 2007. 


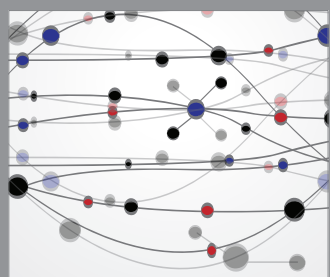

The Scientific World Journal
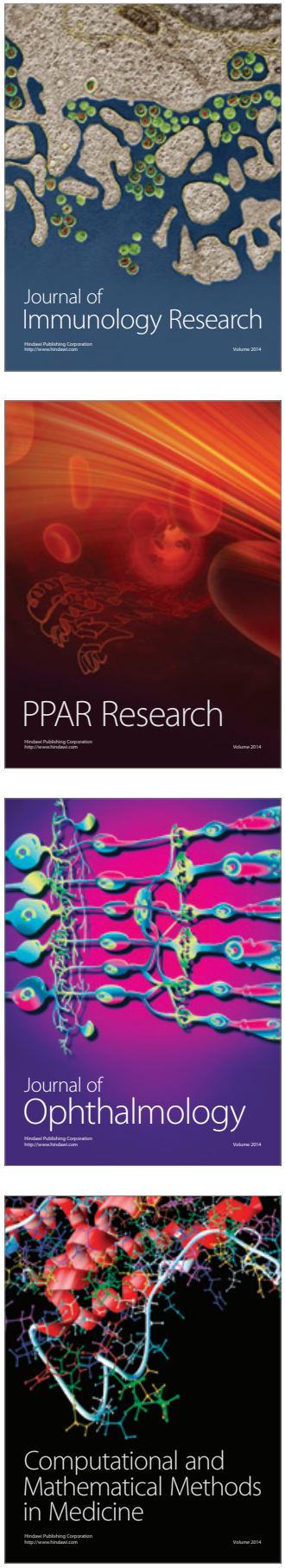

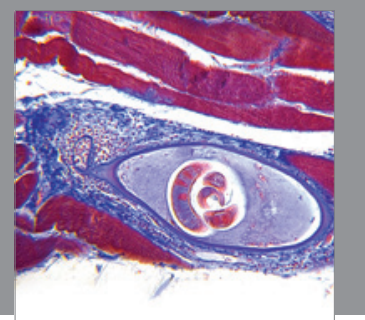

Gastroenterology

Research and Practice
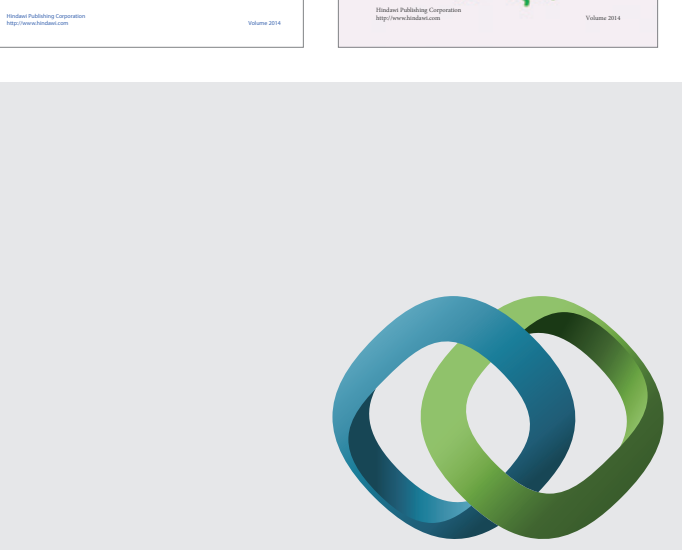

\section{Hindawi}

Submit your manuscripts at

http://www.hindawi.com
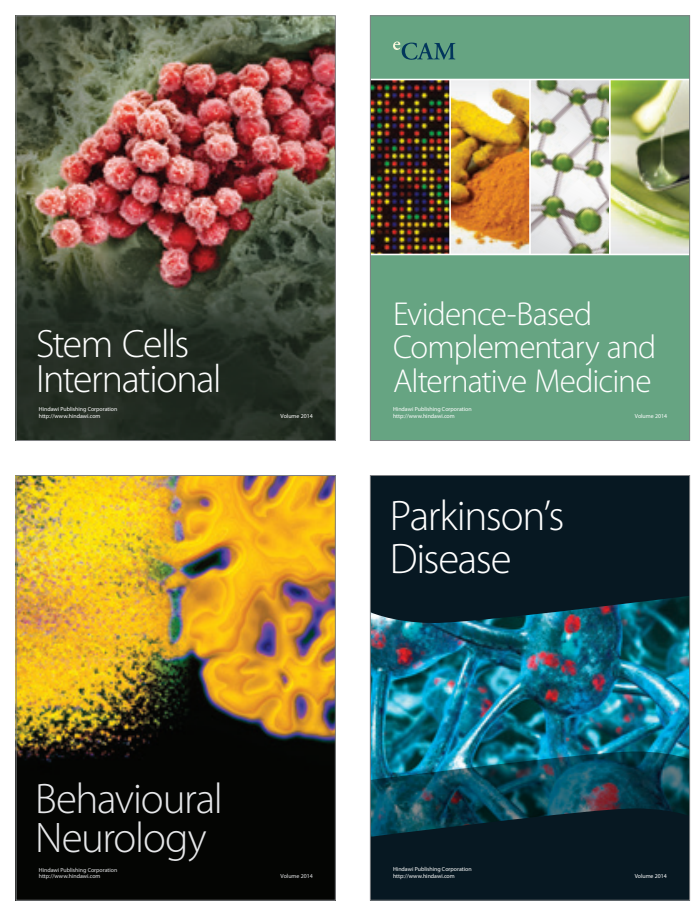

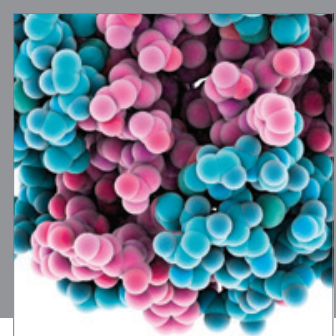

Journal of
Diabetes Research

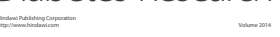

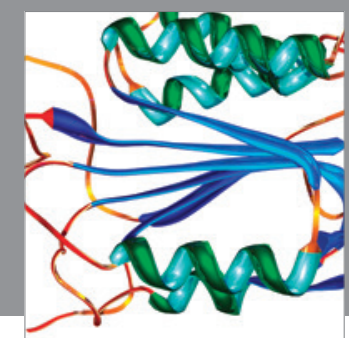

Disease Markers
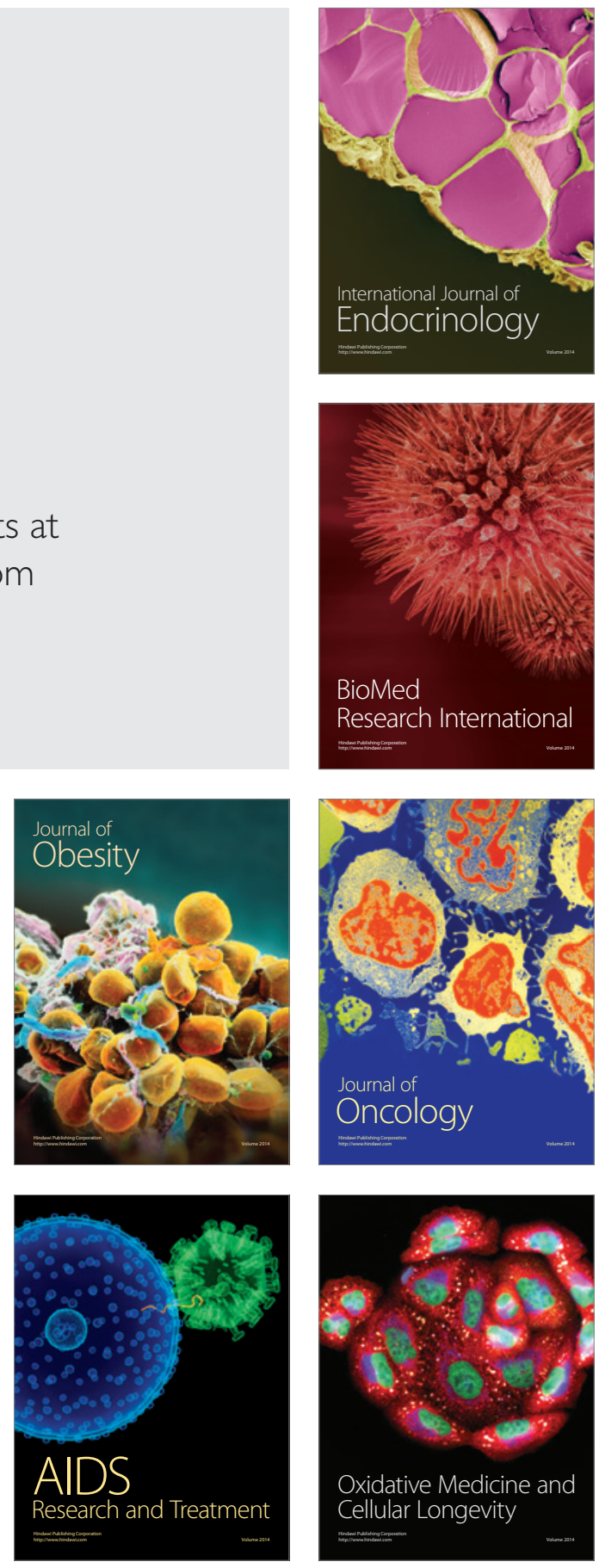